\title{
Sistema de carga inalámbrica para vehículos eléctricos en reposo
}

\section{Wireless charging system for static electric vehicles}

\author{
Iván Yánez ${ }^{1}$
}

\section{Resumen}

El lento crecimiento del uso de vehículos eléctricos se debe a múltiples problemas, uno de ellos es la carga del auto utilizando cable, método conocido como plug-in. Una alternativa tecnológica que puede sustituir a los cables es el sistema de transferencia de energía mediante inducción electromagnética; por el momento esta transferencia de energía es de baja potencia comparada con el sistema por cable. El objetivo de este proyecto de investigación es demostrar que el sistema de carga por inducción es totalmente factible para un vehículo estacionado. Para ello se usa una bobina fuente ubicada en el piso del parqueadero, y una bobina captadora localizada en el bastidor del auto. En este trabajo se demuestra experimentalmente cómo varía la potencia de transferencia al variar las distancias entre las dos bobinas; se consideran tres alternativas: bobinas con núcleos de aire, bobinas con núcleo de hierro común y bobinas con núcleos de hierro separados. Las mediciones se realizan en un prototipo formado por dos bobinas separadas una distancia d y provisto de una escala de medida en milímetros. Finalmente, se evalúa el comportamiento de la energía transferida en función de la distancia entre bobinas y las tres alternativas de núcleo magnético.

\section{Palabras clave}

Carga inalámbrica; sistema inductivo.

\begin{abstract}
The use of electric vehicles expands slowly due to many problems; one of them is charging the car using cable, a method known as plug-in. A technological alternative that can replace cables is the system of energy transfer by electromagnetic induction; for the moment this energy transfer is low power compared to the cable system. The objective of this research project is to demonstrate that the induction charging system is totally feasible for a parked vehicle. It uses a source coil located on the floor of the parking lot, and a pickup coil located in the frame of the car. This paper experimentally demonstrates how the power of transfer varies when the distances between the two coils vary too; three alternatives are considered: coils with cores of air, coils with common iron core and coils with separated iron cores.
\end{abstract}

The measurements are made in a prototype formed by two coils separated by a distance $d$ and provided with a scale of measurement in millimeters. Finally, the behavior of the transferred energy is evaluated according to the distance between coils and the three magnetic core alternatives.

\section{Keywords}

Wireless charging; inductive system.

\section{Introducción}

El Vehículo Eléctrico (EV) es un medio de transporte de personas o carga que utiliza un motor eléctrico como propulsor para el funcionamiento del motor eléctrico; el vehículo usa la energía almacenada en las baterías (Larminie, 2012), cuando las baterías se agotan existen algunas alternativas para cargarlas, entre ellas está el cargarlas mediante cable o por medio de medios inalámbricos, esta última alternativa se investiga en el presente artículo.

El problema que se presenta al cargar las baterías es que durante el proceso el vehículo está conectado por medio de un cable a la pared, en el presente trabajo se plantea la opción 
de usar la inducción electromagnética como alternativa para transferir energía eléctrica al vehículo sin usar cables. Para aplicar el principio de inducción electromagnética, se necesita un núcleo de hierro común entre dos bobinas, la primaria (fuente) y la secundaria (captador). En el caso de aplicar a vehículos eléctricos, esto no es posible, en principio, porque la fuente de carga estaría en el piso, y el receptor de carga está en el bastidor, por tanto se plantean tres alternativas para el núcleo de las bobinas: núcleo de aire, núcleo de hierro común y núcleo de hierro separados, además la medición de voltaje inducido se hace para varias distancias, se usa un prototipo que simula una bobina fuente primaria (piso) y una bobina captadora secundaria (bastidor del auto), para facilitar la medición las dos bobinas tienen una separación regulable.

El sistema de carga que se va a probar se basa en el principio de inducción electromagnética, que consiste en producir electricidad en un conductor debido al movimiento del campo magnético de otro conductor cercano (Bijalwan, 2014), estos principios son bastante conocidos y se los conoce desde la época de Faraday (Geddes, 1981) hasta los experimentos de Tesla en 1900, los sistemas de carga inalámbrica utilizando bobinas que resuenan entre sí han evolucionado, actualmente se cuenta con sistemas que llegan a potencias de transferencia de más de 100 KW (Miśkiewicz, 2017), en el trabajo de investigación presentado en este artículo se investiga la transferencia de energía por inducción en el aire, existen otros trabajos que analizan la transferencia inductiva en el agua (Shi, 2014).

Un campo magnético se lo puede representar mediante líneas de campo, existen dos polos Norte y Sur, y las líneas viajan del polo Norte hacia el Sur. El flujo magnético se lo describe mediante la relación (Serway, 2015):

$$
\phi_{B}=\int B \cdot d S
$$

B es la intensidad del campo magnético, S la superficie por la que atraviesa el campo. El flujo magnético cambia en el espacio, según la distancia que se encuentre entre el polo Norte con el polo Sur, por ello se utiliza un material ferro magnético para evitar la dispersión de las líneas de campo magnético, por ejemplo, en los transformadores, Figura 1.

Existen varios métodos de carga, tanto comerciales como en etapa de prueba; para baja potencia existe el denominado Highly Resonant Wireless Power Transfer, el cual transporta el flujo magnético mediante ondas resonantes (Morris, 2013). Esto ya lo demostró Tesla por medio del circuito resonador básico R-L-C (Marck, 2015). Otro método de transporte de energía son las microondas, sin embargo, por la densidad alta de energía está observado por un estándar de comunicación y no se usa en Vehículos Eléctricos (Xiao, 2014).

Actualmente, los sistemas de carga Wireless se usan ampliamente en todo el mundo, los estándares son aplicados en muchas industrias (Joehren Michael, 2014). La fuerza electromagnética generada en el captador viene dada por la Ley de Faraday, que en forma diferencial se escribe:

$$
\nabla \mathrm{X} E=-\frac{\partial B}{\partial t}
$$

Esta ecuación forma parte de las conocidas Leyes de Maxwell. Para el presente estudio la ecuación anterior se puede reducir a la siguiente, según el número de vueltas de la bobina primaria. 
$V=-N \frac{\partial \phi}{\partial t}$

Donde $V$ es la tensión inducida por una bobina de $\mathrm{N}$ vueltas, y el diferencial es la variación del flujo magnético.

Figura 1. Líneas de campo

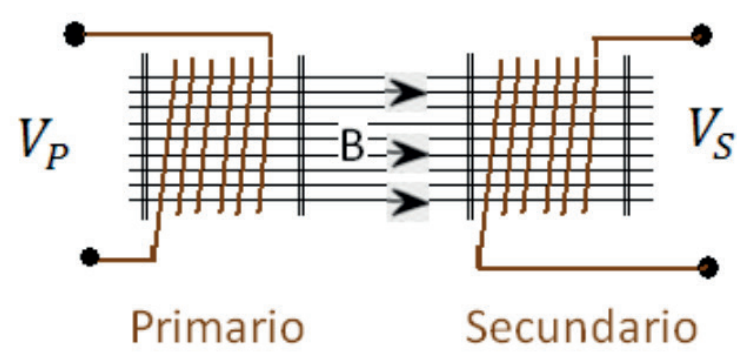

El objetivo general es medir el voltaje inducido en la bobina captadora $\left(V_{c}\right)$ cuando se varía la distancia entre la bobina fuente y la bobina captadora usando tres alternativas de núcleos magnéticos y ver la posibilidad de su aplicación en la carga inalámbrica de vehículos eléctricos. En este trabajo la bobina fuente es la bobina primaria ( $p$ ) y la bobina captadora es la bobina secundaria(s). La relación teórica del transformador (m), viene dada por:

$\frac{N_{p}}{N_{s}}=\frac{V_{p}}{V_{s}}=\frac{I_{s}}{I_{p}}=m$

Cuando los núcleos de la bobina primaria (fuente) y secundaria (captadora) están separados una cierta distancia, el rendimiento de la transferencia por inducción es ß.

$\beta=\frac{V c \max }{V f}$

Donde Vcmax es el máximo voltaje inducido en la bobina captadora y Vf es el voltaje de red aplicado a la bobina fuente. Las mediciones de transferencia inductiva para bobinas con núcleos de hierro separados se las presentó en un primer trabajo (Yánez, 2017);en el presente trabajo se ha complementado con las mediciones para bobinas con núcleos de aire y bobinas con núcleo común. Es importante decir que en las pruebas realizadas en la presente investigación el máximo rendimiento en la transferencia se lo obtuvo con núcleo de hierro común, este acoplamiento tuvo la eficiencia de transferencia de $68 \%$ a $5 \mathrm{~mm}$, en contraste en el artículo de Wen Haibing (Haibing, 2018) se tiene un rendimiento del 78 \% con esa separación.

\section{Materiales, métodos y procedimientos}

El método a usar es experimental, el prototipo de transferencia de carga por inducción se lo validará para diferentes distancias entre la fuente y el captador de energía a través de una separación regulable, Figura 2. 
La bobina fuente tiene $\mathrm{Nf}$ vueltas, y la bobina captadora tiene Nc vueltas de alambre de cobre, se utilizaron dos bobinas con igual número de espiras Nf=Nc. Se realizarán mediciones de transferencia de energía en cuatro alternativas de núcleo magnético.

Prueba 1, núcleo de aire-aire: Medir la transferencia de energía inductiva con núcleo de aire la fuente y núcleo de aire la captadora.

Prueba 2, núcleo de hierro-aire: Medir la transferencia de energía inductiva con núcleo de hierro la fuente y núcleo de aire la captadora.

Prueba 3, núcleo de hierro común: Medir la transferencia de energía inductiva si las dos bobinas comparten el núcleo de hierro.

Prueba 4, núcleo de hierro independiente: Medir la transferencia de energía inductiva si las dos bobinas tienen núcleos de hierro separados.

La bobina captadora tiene un mecanismo de desplazamiento frente a una regla graduada en mm, Figura 2.

Figura 2. Prototipo para medir la potencia transmitida

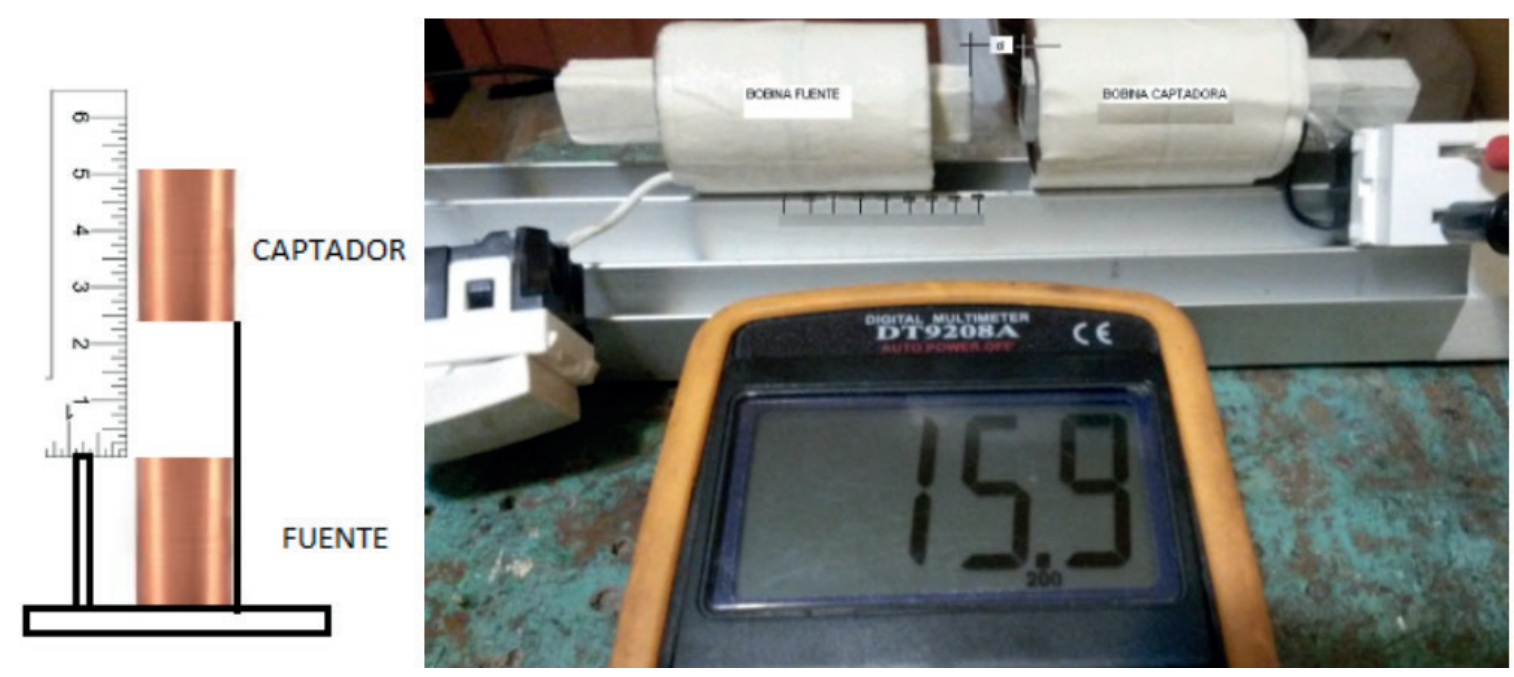

\section{Resultados y discusión}

El voltaje inducido en la captadora (Vc) no es $100 \%$ efectivo, a lo mucho se obtiene un rendimiento en la transferencia de ß, ver ecuación [5].

$\beta=\frac{V c \max }{V f} 100 \%$

Se espera por tanto determinar el rendimiento de transferencia de energía por inducción(ß). Parámetros iníciales medidos:

Voltaje de Red $(\mathrm{Vr}=\mathrm{Vf})$ :

Resistencia bobina fuente:

Resistencia bobina Captadora:
$127.5 \mathrm{~V}$

$9.8 \mathrm{~K} \Omega$

$9.9 \mathrm{~K} \Omega$ 
Se procede a medir el voltaje para diferentes separaciones entre bobinas, para cada distancia se mide cuatro veces el voltaje y se calcula el promedio, para obtener el rendimiento de transferencia inalámbrica de carga.

\subsection{Prueba de transferencia inductiva con núcleo de aire la fuente y la captadora}

Para esta prueba no se utilizan núcleos ferro magnéticos en las bobinas, se hacen las mediciones con núcleo de aire. Los resultados de la medición de voltaje en la bobina captadora se presentan en la Tabla 1.

Tabla 1. Medición de voltaje en la bobina captadora

\begin{tabular}{|c|c|c|c|c|c|c|c|}
\hline $\begin{array}{c}\text { Distancia } \\
\mathbf{d}(\mathbf{m m})\end{array}$ & $\mathbf{V 1}$ & $\mathbf{V 2}$ & $\mathbf{V 3}$ & $\mathbf{V 4}$ & $\begin{array}{c}\text { Voltaje } \\
\text { promedio } \\
\mathbf{( V )}\end{array}$ & $\mathbf{\sigma}$ & $\begin{array}{c}\boldsymbol{B}=\mathbf{V} / \mathbf{V r} \mathbf{x} \mathbf{1 0 0} \\
\%\end{array}$ \\
\hline 0 & 1.33 & 1.23 & 1.28 & 1.24 & 1.3 & 0.045 & 1.0 \\
\hline 5 & 0.90 & 0.96 & 0.90 & 0.94 & 0.9 & 0.030 & 0.7 \\
\hline 10 & 0.67 & 0.64 & 0.60 & 0.64 & 0.6 & 0.029 & 0.5 \\
\hline 15 & 0.47 & 0.45 & 0.42 & 0.48 & 0.5 & 0.026 & 0.4 \\
\hline 20 & 0.37 & 0.30 & 0.32 & 0.36 & 0.3 & 0.033 & 0.3 \\
\hline 25 & 0.25 & 0.26 & 0.24 & 0.28 & 0.3 & 0.017 & 0.2 \\
\hline 30 & 0.17 & 0.17 & 0.18 & 0.22 & 0.2 & 0.024 & 0.1 \\
\hline 35 & 0.16 & 0.13 & 0.13 & 0.17 & 0.1 & 0.021 & 0.1 \\
\hline 40 & 0.14 & 0.09 & 0.11 & 0.14 & 0.1 & 0.024 & 0.1 \\
\hline
\end{tabular}

\subsection{Prueba de transferencia inductiva con núcleo de hierro la fuente y con núcleo de aire la captadora}

En esta prueba se utiliza núcleo ferro magnético en la bobina fuente (piso), la bobina captadora (auto) tiene núcleo de aire. Los resultados de medición de voltaje en la Bobina captadora se presentan en la Tabla 2.

Tabla 2. Medición de voltaje en la bobina captadora

\begin{tabular}{|c|c|c|c|c|c|c|c|}
\hline $\begin{array}{c}\text { Distancia d } \\
(\mathbf{m m})\end{array}$ & V1 & V2 & V3 & V4 & $\begin{array}{c}\text { Voltaje } \\
\text { promedio } \\
\text { (Vc) }\end{array}$ & $\sum$ & $\begin{array}{c}\text { B=Vc/Vr } \mathbf{x} \\
\mathbf{1 0 0} \%\end{array}$ \\
\hline 0 & 5.90 & 5.91 & 5.96 & 5.85 & 5.9 & 0.045 & 4.6 \\
\hline 5 & 4.40 & 4.37 & 4.04 & 4.00 & 4.2 & 0.212 & 3.3 \\
\hline 10 & 3.02 & 3.02 & 2.92 & 2.96 & 3.0 & 0.049 & 2.3 \\
\hline 15 & 2.22 & 2.22 & 2.16 & 2.12 & 2.2 & 0.049 & 1.7 \\
\hline 20 & 1.72 & 1.64 & 1.64 & 1.61 & 1.7 & 0.047 & 1.3 \\
\hline 25 & 1.37 & 1.28 & 1.28 & 1.28 & 1.3 & 0.045 & 1.0 \\
\hline 30 & 1.05 & 1.01 & 1.01 & 1.01 & 1.0 & 0.020 & 0.8 \\
\hline 35 & 0.83 & 0.81 & 0.83 & 0.82 & 0.8 & 0.010 & 0.6 \\
\hline 40 & 0.69 & 0.67 & 0.68 & 0.66 & 0.7 & 0.013 & 0.5 \\
\hline
\end{tabular}




\subsection{Prueba de transferencia inductiva con núcleo de hierro común}

En esta prueba la bobina fuente y la bobina captadora tienen núcleo común ferro magnético. Los resultados de medición de voltaje en la bobina captadora se presentan en la Tabla 3.

Tabla 3. Medición de voltaje en la bobina captadora

\begin{tabular}{|c|c|c|c|c|c|c|c|}
\hline $\begin{array}{c}\text { Distancia } \\
\mathbf{d}(\mathbf{m m})\end{array}$ & V1 & V2 & V3 & V4 & $\begin{array}{c}\text { Voltaje promedio } \\
(\mathbf{V c})\end{array}$ & $\sum$ & $\begin{array}{c}\text { B=Vc/Vr } \mathbf{x} \\
\mathbf{1 0 0} \%\end{array}$ \\
\hline 0 & 85.6 & 87.2 & 87.1 & 86.8 & 86.7 & 0.737 & 68.0 \\
\hline 5 & 79.9 & 82.8 & 82.5 & 82.7 & 82.0 & 1.389 & 64.3 \\
\hline 10 & 74.2 & 78.1 & 77.4 & 77.0 & 76.7 & 1.711 & 60.1 \\
\hline 15 & 68.2 & 72.5 & 71.7 & 71.4 & 71.0 & 1.891 & 55.6 \\
\hline 20 & 63.4 & 67.4 & 66.4 & 66.3 & 65.9 & 1.723 & 51.7 \\
\hline 25 & 58.9 & 62.4 & 60.7 & 61.6 & 60.9 & 1.503 & 47.8 \\
\hline 30 & 53.7 & 57.1 & 56.4 & 56.7 & 56.0 & 1.544 & 43.9 \\
\hline 35 & 46.7 & 51.6 & 52.3 & 52.2 & 50.7 & 2.685 & 39.8 \\
\hline 40 & 44.6 & 46.8 & 47.1 & 47.4 & 46.5 & 1.274 & 36.5 \\
\hline
\end{tabular}

\subsection{Prueba de transferencia inductiva con núcleos de hierro separados}

En esta prueba la bobina fuente y la bobina captadora tienen núcleo de hierro, pero están separados. Los resultados de medición de voltaje en la Bobina captadora se presentan en la Tabla 4.

Tabla 4. Medición de voltaje en la bobina captadora

\begin{tabular}{|c|c|c|c|c|c|c|c|}
\hline $\begin{array}{c}\text { Distancia d } \\
(\mathbf{m m})\end{array}$ & V1 & V2 & V3 & V4 & $\begin{array}{c}\text { Voltaje promedio } \\
(\mathbf{V c})\end{array}$ & $\boldsymbol{\Sigma}$ & B=Vc/Vr $\mathbf{x} \mathbf{1 0 0} \%$ \\
\hline 0 & 52.8 & 53.2 & 53.2 & 52.6 & 53.0 & 0.300 & 41.6 \\
\hline 5 & 24.7 & 23.1 & 23.4 & 23.0 & 23.6 & 0.785 & 18.5 \\
\hline 10 & 16.2 & 15.7 & 16.2 & 15.8 & 16.0 & 0.263 & 12.5 \\
\hline 15 & 11.9 & 11.6 & 11.4 & 11.2 & 11.5 & 0.299 & 9.0 \\
\hline 20 & 9.0 & 8.7 & 8.8 & 8.5 & 8.8 & 0.208 & 6.9 \\
\hline 25 & 7.1 & 6.8 & 6.8 & 6.7 & 6.9 & 0.173 & 5.4 \\
\hline 30 & 5.6 & 5.5 & 5.5 & 5.4 & 5.5 & 0.082 & 4.3 \\
\hline 35 & 4.5 & 4.4 & 4.3 & 4.3 & 4.4 & 0.096 & 3.5 \\
\hline 40 & 3.6 & 3.6 & 3.6 & 3.6 & 3.6 & 0.000 & 2.8 \\
\hline
\end{tabular}

\subsection{Discusión}

A continuación, se presenta las gráficas del voltaje promedio inducido en la bobina captadora para los tres tipos de núcleos, las curvas son decrecientes, Figura 3. 
Figura 3. Disminución de la transferencia de energía en función de la distancia entre bobinas para tres tipos de núcleos

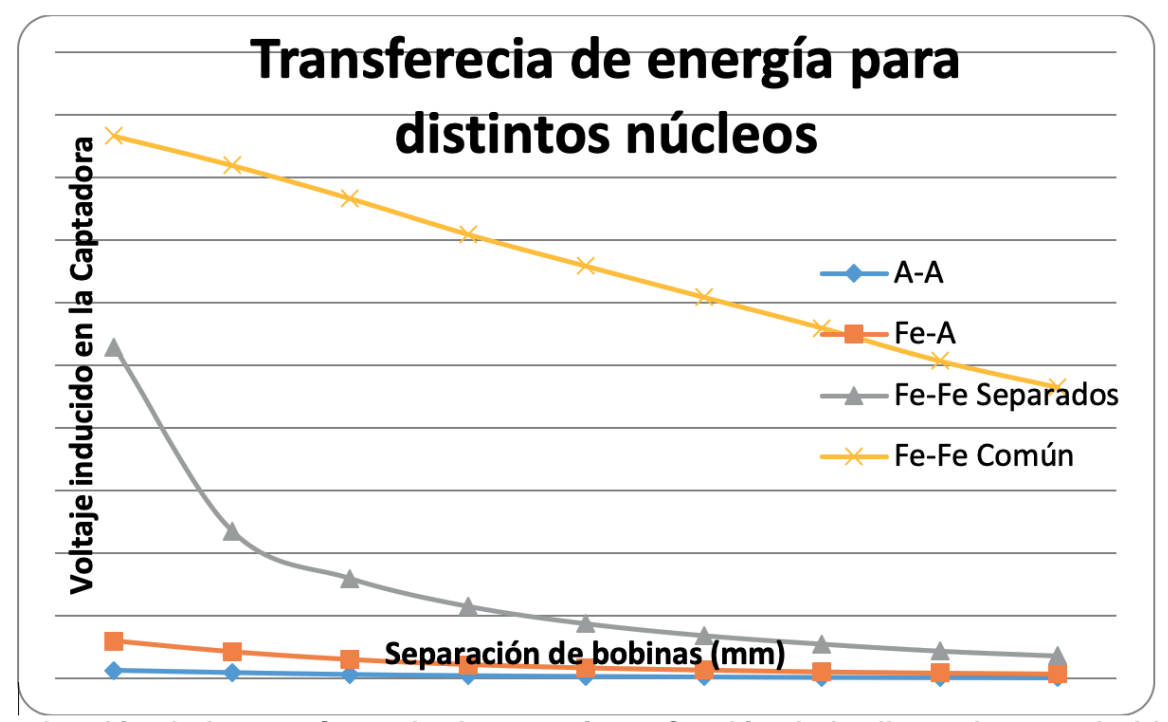

Para el caso de núcleos de hierro separados, al realizar una interpolación a los datos obtenidos de las mediciones, usando Excel se determina que la función matemática que más se aproxima a la tendencia de puntos medida es de tipo exponencial decreciente, Figura 4.

Figura 4. Modelo matemático por interpolación Fe-Fe separados

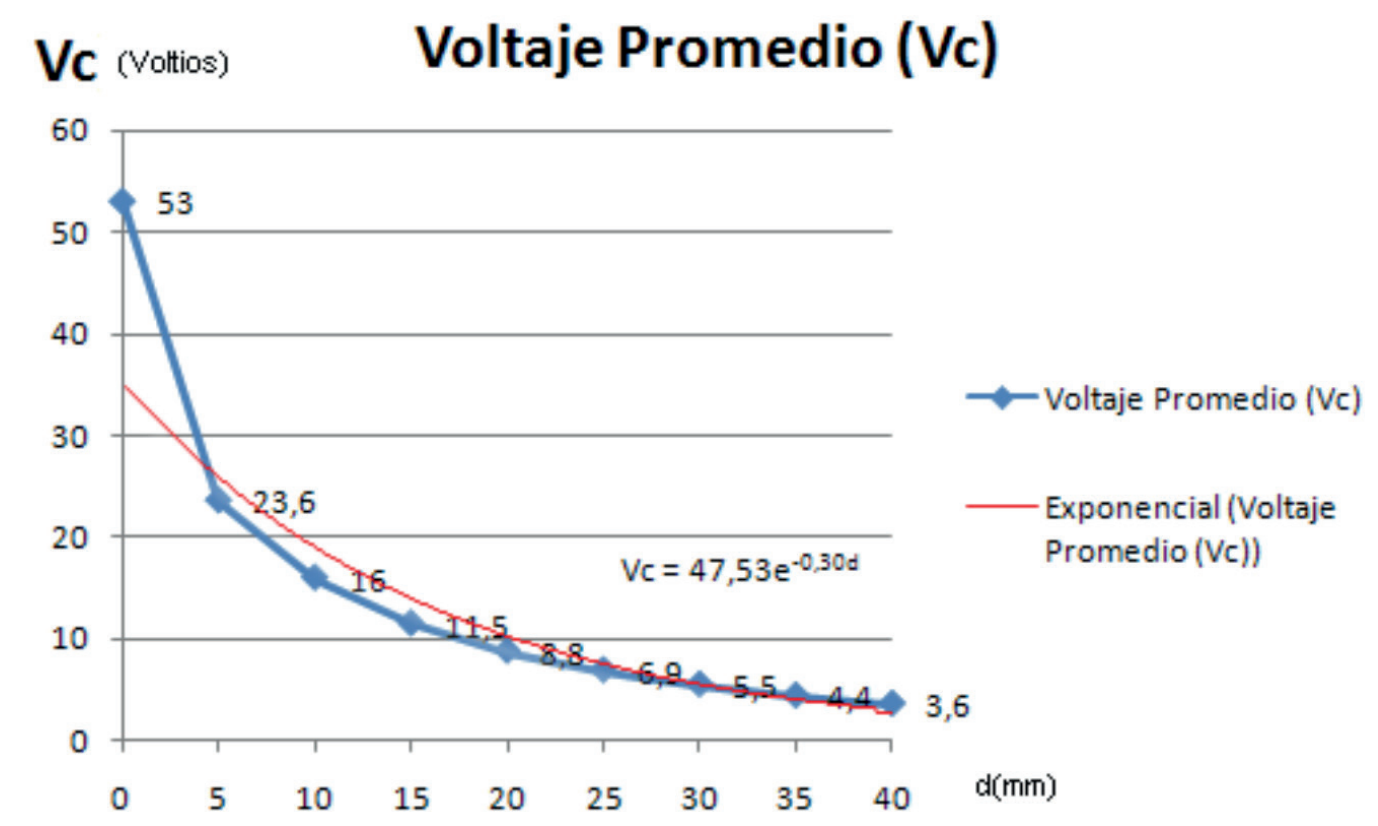

Y la función matemática obtenida por interpolación para la transferencia inductiva es:

$V c=47,53 e^{-0,30 d}$ 
Para el caso de bobinas con núcleo común de hierro, al realizar una interpolación a los datos obtenidos de las mediciones, se determina que la función matemática que más se aproxima a la tendencia de puntos de medida es una recta de pendiente negativa, Figura 5.

Figura 5. Modelo matemático por interpolación Fe-Fe común

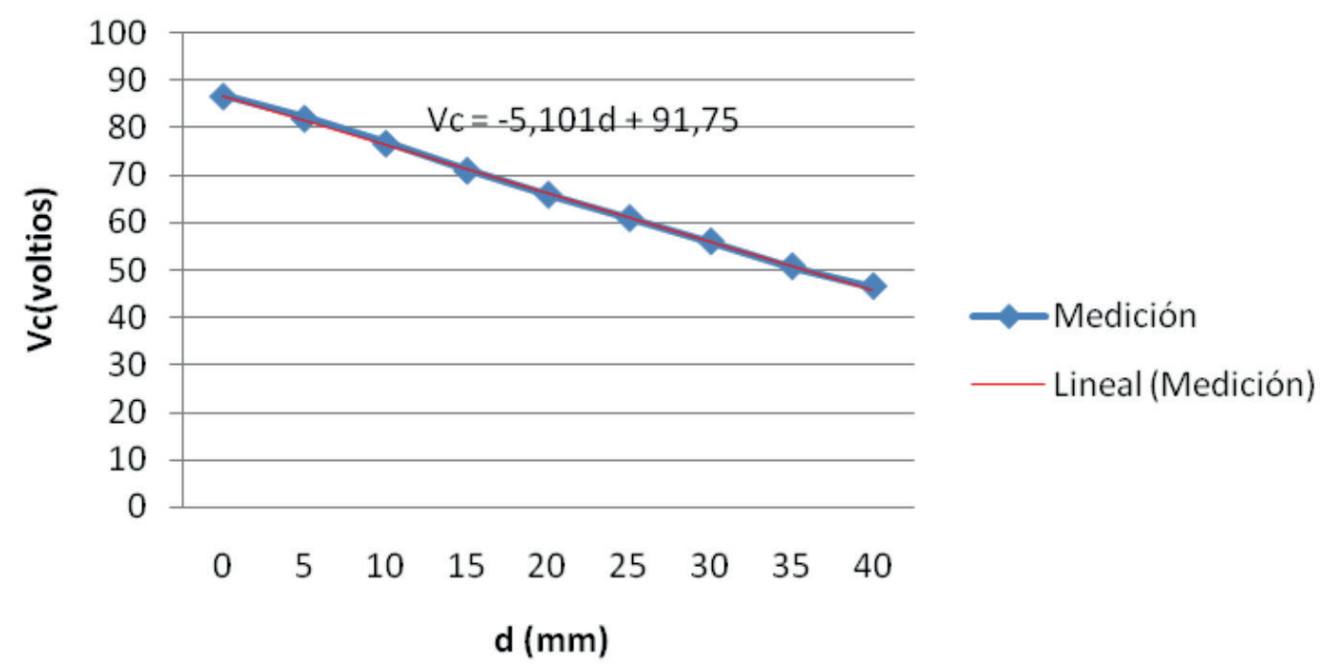

Y la función matemática obtenida por interpolación para la transferencia inductiva es:

$$
V c \equiv 5.101 d+91.75
$$

Luego de realizar las mediciones respectivas se determina que el método de carga inalámbrico es factible si la bobina fuente que está en el piso se encuentra lo más cerca posible de la captadora, se sugiere un mecanismo retráctil para regular la distancia. Para aplicar al vehículo se debe usar un mecanismo retráctil automático que asegure que las bobinas fuente y captadora estén acopladas en el momento de la carga. Cuando el vehículo se pone en marcha el mecanismo se retrae. Existen algunas innovaciones que logran el acoplamiento, por ejemplo, en el artículo denominado "Inductive Power Transfer System Integration for Battery-Electric Vehicles"(Lorico, 2011) se presenta una alternativa tecnológica que permite reducir el paquete de baterías hasta un $48 \%$, denominada ICPT que es un singular tipo de acoplamiento inductivo. En la actualidad, compiten distintos sistemas de carga, tanto alámbricos como inalámbricos, siendo los sistemas inductivos los que mayor capacidad de transferencia tienen incluso si el vehículo está en movimiento, en el artículo denominado "Inductive Energy Transfer for Electric Vehicles" (Nietschke, 2011) se discuten algunas alternativas de carga inductiva. Los sistemas inductivos tradicionales han sido mejorados mediante la introducción de campos magnéticos resonantes, una descripción detallada de este sistema se lo puede ver en el artículo denominado "Standard Proposal for Resonant Inductive Charging of Electric Vehicles" (Mathar, 2012). Eliminar cables es una ventaja para el usuario del vehículo porque su uso engloba muchos problemas como el deterioro, olvidar que el auto está enchufado entre otros, con el propósito de aumentar la potencia de transferencia inductiva el autor del artículo denominado "Compact, Safe and Efficient Wireless and Inductive Charging for Plug-In Hybrids and Electric Vehicles" (Körner, 2014) plantea como alternativa el aumento del campo magnético, conjuntamente con el diseño de un sistema electrónico que verifique permanentemente la presencia en el entorno 
del sistema de objetos extraños. Conjuntamente con los modelos resonantes, casi todas las soluciones de transferencia de carga inductiva están planteadas en el dominio de la frecuencia, un análisis detallado de las pruebas del modelo matemático se presenta en el artículo "Coupled Heat-Electromagnetic Simulation of Inductive Charging Stations for Electric Vehicles" (Kaufmann, 2012).

Sin duda, el futuro de la transferencia inductiva de carga está orientado a vehículos que no necesariamente están en reposo, cuando los vehículos están en movimiento los problemas se multiplican y la eficiencia de transferencia cae drásticamente, por ello se han ideado sistemas de monitoreo electrónico, por ejemplo, en el artículo denominado "Electro-Optical System for Evaluation of Dynamic Inductive Wireless Power Transfer to Electric Vehicles" (Cardoso, 2019), se presenta un sistema óptico de posicionamiento del auto sobre las bobinas fuentes y se mide la eficiencia de transferencia. Casi todas las soluciones de transferencia utilizan bobinas con espirales denominadas de Arquímedes, y los modelos de transferencia se los pone a prueba utilizando métodos de elementos finitos como se demuestra en el artículo "Analytical design of Archimedean spiral coils used in inductive power transfer for electric vehicles application" (Aditya, 2018). Generalmente para realizar la transferencia inductiva se utilizan sistemas monofásicos y trifásicos, en la actualidad los fabricantes de sistemas de carga se ponen de acuerdo en los estándares de carga para sistemas con cables y sin cables, en el artículo denominado "Charging Architectures for Electric and Plug-In Hybrid Electric Vehicles" (Rivera, 2016) se presenta un excelente análisis de las distintas configuraciones de carga y los estándares utilizados. Probablemente la potencia de transferencia no sea la misma para distintos vehículos por lo que será necesario tener una capacidad para ajustar el voltaje de salida, por ejemplo, en el artículo "Wireless power transfer receiver with adjustable coil output voltaje for multiple receivers application" (Ahn, 2019) se plantea un sistema de transferencia de carga con múltiples salidas.

Finalmente se prevé que el futuro de la carga de vehículos eléctricos está en los sistemas inductivos, no obstante para que esto funcione se debe automatizar el proceso de carga, de manera que el usuario no interactúe con el sistema, algunas alternativas de automatización se presentan en el artículo "Automated robot-based charging system for electric vehicles" (Walzel, 2016), para lograr la máxima eficiencia en la transferencia de energía por el método inductivo con núcleo común ferro magnético, se necesita un mecanismo automatizado retráctil.

\section{Conclusiones y recomendaciones}

Del estudio se presentan a continuación las siguientes conclusiones:

El sistema de carga inalámbrica, por el método inductivo, se encuentra formado por dos bobinas una fuente y una captadora, La bobina fuente está en el piso y la captadora en el bastidor del vehículo, siendo el núcleo entre bobinas un factor determinante en la transferencia inductiva de energía.

El rendimiento del sistema de transferencia inductivo conformado por bobinas con núcleo de aire es aproximadamente $\beta=1 \%$.

Cuando se tiene la bobina fuente con núcleo de hierro, y la captadora con núcleo de aire, el rendimiento sube al $ß=4.6 \%$. Para este caso, se ha hecho la prueba con un núcleo de aire en la bobina del vehículo con el fin de no aumentar el peso del mismo. Mediante algún diseño en la captadora se puede hacer que comparta el núcleo de la bobina fuente, esto lo tenemos en el caso de bobina con núcleo de hierro común. 
Si la bobina fuente y la bobina captadora comparten un núcleo de hierro común, el rendimiento mejora en gran manera llegando al significativo valor de $\beta=68 \%$. Hay que observar que para este caso la potencia transferida es directamente proporcional a la separación de las bobinas, a pesar de que el núcleo de hierro es el mismo para ambas. Para este caso se debe diseñar la bobina del auto de tal manera que mediante un mecanismo retráctil se ubique en el núcleo de hierro de la bobina fuente ubicada en el piso.

En la última prueba tanto la bobina fuente como la captadora tienen núcleo de hierro, y están separadas una distancia variable. Para este caso el rendimiento en la transferencia es de $\beta=41,6 \%$.

En las mediciones, la máxima dispersión es $\sigma=2.685$, que es la desviación estándar mayor y se produjo en el caso de núcleos de hierro común.

De acuerdo a los resultados obtenidos, el mayor rendimiento de transferencia se produce para bobinas con núcleo de hierro común, por tanto se debe diseñar un mecanismo de acoplamiento que permita introducir el núcleo de hierro de la bobina fuente dentro de la bobina captadora. Este sistema de acoplamiento debe ser automático, y se sugiere debe estar en la bobina fuente, en el piso o la pared. Se recomienda una patente para esta innovación.

\section{Bibliografía}

Ahn, D. (2019). Wireless power transfer receiver with adjustable coil output voltage for multiple receivers application. IEEE Transactions on Industrial Electronics, 4003-4012.

Bijalwan, T. (2014). Review paper based on wireless charging. ISTP Journal, 1.

Cardoso, L. A. (2019). Electro-Optical System for Evaluation of Dynamic Inductive Wireless Power Transfer to Electric Vehicles. International Conference on Green Energy and Networking, 154-174.

Geddes. (1981). Advanced Physics. Londres: Palgrave.

Haibing, W. (2018). A novel electromagnetic actuator in an inductive power transmission system for autonomous underwater vehicle. Advances in Mechanical Engineering, 1-6.

Joehren, M. (2014). Development of an optimized wireless charging application. NXP Semiconductors, January.

Körner, A. (2014). Compact, Safe and Efficient Wireless and Inductive Charging for Plug-In Hybrids and Electric Vehicles. Advanced Microsystems for Automotive Applications 2014, 213-234.

Larminie. (2012). Electric Vehicle Technology, Explained. Nueva Jersey: WILEY.

Lorico. (2011). Inductive Power Transfer System Integration for Battery-Electric Vehicles. Sustainable Automotive Technologies 2011, 75-83.

Mathar. (2012). Standard Proposal for Resonant Inductive Charging of Electric Vehicles. Advanced Microsystems for Automotive Applications 2012, 57-68.

Nietschke, W. (2011). Inductive Energy Transfer for Electric Vehicles. ATZ worldwide e Magazine, 22-27.

Kaufmann, C. (2012). Coupled Heat-Electromagnetic Simulation of Inductive Charging Stations for Electric Vehicles. Progress in Industrial Mathematics at ECMI 2012, 27-36.

Aditya, K. (2018). Analytical design of Archimedean spiral coils used in inductive power transfer for electric vehicles application. Electrical Engineering, 1819-1826.

Rivera, S. (2016). Charging Architectures for Electric and Plug-In Hybrid Electric Vehicles. Technologies and Applications for Smart Charging of Electric and Plug-in Hybrid Vehicles, 111-149.

Marck, E. (2015). The Convenience de Wireless Charging. Media Tek, 8.

Miśkiewicz. (2017). Multithreading Analysis of Properties and Electromagnetic Interference in Inductive Contactless Power Supply System with Bidirectional Energy Flow-Part 1: Topology System for Electric Vehicles. Analysis and Simulation of Electrical and Computer Systems, 161-172. 
Morris, K. (2013). Highly Resonant Wireless Power Transfer. WITRICITY Corporation, 2.

Serway, R. (2015). Física para las Ciencias e Ingeniería. Ciudad de México, México: Paraninfo.

Shi, J.-g. (2014). Design and analysis of an underwater inductive coupling power transfer system for autono-

mous underwater vehicle docking applications. Journal of Zhejiang University SCIENCE C, 51-62.

Walzel, B. (2016). Automated robot-based charging system for electric vehicles. Internationales Stuttgarter Symposium, 937-949.

Xiao, L. (2014). Wireless Charger Networking for Mobile Devices. Wireless Charger Networking for Mobi.

Yánez, I. (2017). Sistema de carga inalámabrico para vehículos eléctricos en reposo. Memorias I Congreso de Ingeniería UTE Santo Domingo de los Tsachilas, --. 